\title{
Zeina Fani Alpi, Dévotions lapidaires : reliefs divins du
}

\section{Liban romain}

Beyrouth, Ministère de la culture, Direction générale des Antiquités

(BAAL hors-série XI), 2016, pp. 344, ISSN:1683-0083, LL 30.000

\section{Corinne Bonnet}

\section{(2) OpenEdition}

Journals

Édition électronique

URL : http://journals.openedition.org/mythos/1139

DOI : $10.4000 /$ mythos. 1139

ISSN : 2037-7746

Éditeur

Salvatore Sciascia Editore

Référence électronique

Corinne Bonnet, «Zeina Fani Alpi, Dévotions lapidaires : reliefs divins du Liban romain », Mythos [En ligne], 13 | 2019, mis en ligne le 01 décembre 2019, consulté le 24 septembre 2020. URL : http:// journals.openedition.org/mythos/1139; DOI : https://doi.org/10.4000/mythos.1139

Ce document a été généré automatiquement le 24 septembre 2020.

Mythos 


\title{
Zeina Fani Alpi, Dévotions lapidaires : reliefs divins $d u$ Liban romain
}

\author{
Beyrouth, Ministère de la culture, Direction générale des Antiquités
}

(BAAL hors-série XI), 2016, pp. 344, ISSN:1683-0083, LL 30.000

\section{Corinne Bonnet}

\section{RÉFÉRENCE}

Zeina Fani Alpi, Dévotions lapidaires : reliefs divins du Liban romain, Beyrouth, Ministère de la culture, Direction générale des Antiquités (BAAL hors-série XI), 2016, pp. 344, ISSN: 1683-0083, LL 30.000

1 Sous le joli titre de "Dévotions lapidaires", Zeina Fani Alpi propose un recueil exhaustif des reliefs du Liban romain se référant aux dieux, un travail très soigneux, qui examine à la fois les questions de forme et de contenu, avec finesse et sagacité, un ouvrage précieux et attendu qui rendra de grands services aux historiens de l'art, aux archéologues et aux historiens des religions. Rolf Stucky, dans sa courte Préface (p. 5) souligne d'emblée le caractère "narratif » de ces documents figurés, insérés dans un contexte cultuel ; ils servent la communication entre les hommes et les dieux et, selon un langage qui leur est propre, ils racontent une histoire, éclairent l'identité et les pouvoirs des dieux convoqués, l'environnement rituel, tout comme les intentions des dédicants. La variété des reliefs étudiés, 229 en tout, depuis les reliefs ornant les petits autels individuels jusqu'aux reliefs muraux décorant les sanctuaires, commandités par les autorités publiques, permet de toucher à divers registres de la pratique cultuelle. De même la diversité des styles, depuis les représentations assez frustes jusqu'aux images imprégnées de références gréco-romaines, donne à voir tout un éventail de goûts et d'influences culturelles, de stratégies d'affichage de ce que l'on pourrait appeler des « identités » ou des «affiliations ». L'analyse approfondie des reliefs imagés fournit en tout cas bien des éléments de réflexion sur les processus d'" hellénisation » ou d'« acculturation » au Liban romain ; elle conduit aussi à s'interroger sur la circulation 
de modèles ou de traditions entre la côte et la montagne, entre la montage et la Békaa, voire au-delà, en direction de la Palmyèrene et Nabatène. Les enjeux sont donc importants et ils sont abordés tout en nuances, après avoir fixé solidement le socle de la réflexion.

2 Celui-ci est constitué par la première partie du volume : le catalogue (p. 13-239). Pour chacun des 229 reliefs pris en compte, dûment illustrés, avec des photographies de très bonne qualité, l'Auteur fournit une description matérielle de l'objet (fonction, matière, dimensions) et précise son contexte d'origine (souvent un sanctuaire), ainsi que son lieu de conservation. Une datation est proposée, la bibliographie recueillie. Vient ensuite une minutieuse description des images, en particulier des personnages divins représentés. Une identification est avancée lorsque des éléments précis viennent la supporter (par ex. pour le relief 1, Déméter est reconnaissable à la présence d'un épi de blé et d'un pavot; pour le relief 2, Hélios avec sa tête radiée...). Certaines identifications sont incertaines et sont donc dûment discutées par référence aux hypothèses des uns et des autres (par ex. le relief 4 où Mouterde a cru reconnaître la Dea Syria assimilée à Aphrodite Ourania, mais où, en suivant Hajjar, l'auteur identifie plutôt une Tychè coiffée d'un kalathos). Il faut saluer la prudence dont l'Auteur fait preuve dans cet exercice, en présentant les diverses options et en ne tranchant pas quand les éléments font défaut pour asseoir une interprétation de préférence à l'autre. Pour le relief $n^{\circ} 167$, de Kafr Zabad, il semble néanmoins, au vu de l'inscription grecque mentionnant Leukathea que l'on puisse désigner la "déesse encadrée de lions» sous le nom de Leucothéa, dont la figure et le culte ont bien pénétré, sans doute depuis Tyr, dans l'arrière-pays libanais.

3 Le Catalogue, en tous points remarquable, montre une grande richesse de références : Déméter/Cérès, Hélios/Sol, Tychè/Fortuna, Zeus/Jupiter (en particulier Héliopolitain), Kronos/Saturne, Gè, Athéna, Apollon, Artémis/Diane, Aphrodite/Vénus (notamment lugens), Héraclès/Hercule, Arès/Mars, Hermès/Mercure, Dionysos, Pan, Sélénè/Luna, Némésis, Hygie, Mithra, mais aussi Calliopè, Nikè, Eros, Nymphe, Méduse/Gorgone, Persée, Triptolème, Ganymède, satyre, ménade, taureaux, sphinx, etc. Les assemblages de divinités sont spécialement intéressants: ils relèvent d'une logique "panthéonique » qu'il n'est pas toujours aisé de déchiffrer, comme pour le curieux autel octogonal en calcaire ( $\mathrm{n}^{\circ} 151$ du catalogue), provenant de Fakié dans la Békaa nord. Attribué au II siècle de notre ère, il donne à voir sur les huit facettes : Jupiter Héliopolitain, Vénus héliopolitaine, une divinité non identifiée avec certitude, Sol, Luna, Mars, peut-être Allath et Mercure. L'empreinte héliopolitaine est très claire, mais la logique de l'assemblage reste obscure. De même pour l'édicule de Kafr Dan, dans la même région, dont les quatre faces montrent Héraclès/Hercule, un dieu nu (pasteur ?), un agneau couché à ses pieds, une déesse en trône avec kalathos (Vénus héliopolitaine? Atargatis?), Eros. La difficulté est de comprendre si ces différents personnages sont regroupés en fonction d'un horizon cultuel (ils partagent un sanctuaire) ou d'un horizon narratif qui relèverait du champ de la parenté, par exemple, ou du mythe, pour autant que cette catégorie fasse sens ici.

Certaines pièces sont magnifiques et fascinantes, comme le cippe de calcaire $\left(n^{\circ} 183\right)$ provenant de Niha, plus spécifiquement de l'adyton du temple A, qui fait plus d'un mètre de hauteur, avec une Tychè/Fortune placée dans une niche et flanquée de deux énormes lions. Dans un style très hybride, il traduit avec force la puissance de la déesse et les bienfaits qu'on attend d'elle. Du même lieu, est aussi originaire un autel ( $\left.{ }^{\circ} 191\right)$ 
qui montre Jupiter héliopolitain encadré de protomés de taureau, surmontant une niche à l'intérieur de laquelle apparaît une petite figure féminine. L'agencement des deux images est intrigant, mais en l'absence de textes, il n'est pas aisé de comprendre ce qu'il dit des relations entre ces personnages (hiérarchie, protection ?). Il faut en tout cas résister à la tentation de voir un "panthéon» dans toutes ces figures divines attestées en divers lieux, à diverses époques; il convient aussi de considérer que les noms gréco-romains listés ci-dessus sont des « étiquettes » commodes pour décrire ce que nous voyons, ou croyons voir, nous les spécialistes du XXI siècle. Ce que voyait un habitant du Liban romain, ou mieux ce que voyaient les habitants du Liban romain, dont le regard n'était assurément pas univoque, est extrêmement difficile à fixer. Quelques-uns de ces reliefs portent des inscriptions, mais c'est rarement le cas; qui plus est, les dédicaces gravées nous font parfois découvrir des appellations inattendues et inconnues, comme sur le relief $n^{\circ} 189$, ornant un autel du temple B de Niha, qui est offert Deo Hadrani, à un « dieu Hadranès ", dont l'image s'apparente fortement à celle de Jupiter Héliopolitain.

5 La deuxième partie du volume, intitulée «Étude » (p. 243-302), examine les documents réunis sous cinq angles différents : 1 . Les matériaux et supports ; 2 . La technique (taille, outils, techniques particulières, finition); 3. Les formes (pied, buste, mi-corps, frontalité, profil...); 4. L'iconographie. Je n'ai guère les compétences pour me prononcer sur les trois premières parties, mais je note que le calcaire local est le matériau le plus usité, le marbre étant peu attesté tout comme le basalte (p. 243). Intéressante aussi l'observation (p. 268) selon laquelle la nudité est l'apanage des divinités masculines, à l'exception d'une nymphe émergeant de l'eau à Baalbek. La question de la relation entre corps et vêtement permet de noter que certains vêtements mettent en valeur l'anatomie du corps, en accord avec une option anthropomorphique, alors que d'autres couvrent si bien le corps qu'on s'oriente vers un traitement schématique ou géométrique du corps divin (p. 268-269). En matière d'iconographie, Z. Fani Alpi souligne la variété des types: divinités cosmiques, divinités olympiennes, héros divinisé ou héros de la mythologie classique, divinités indigènes, divinités apotropaïques ou protectrices (des cités ou communautés notamment), en lien avec l'agriculture, la vigne, les animaux. Si l'inspiration gréco-romaine est très prégnante, les modèles héliopolitains le sont tout autant, dans un rayon assez étendu. L'Auteur examine au cas par cas les figures majeures, en commençant par les diverses déclinaisons de Zeus/Jupiter, ainsi que les figures qui s'y rapportent (aigles, taureaux...). Viennent ensuite les dieux cosmiques, également très bien représentés. $\mathrm{Z}$. Fani Alpi affirme à juste titre qu'Hélios est bien l'objet d'un culte en tant que tel, sans passer par de prétendus «syncrétismes » (p. 278-279). Hermès/Mercure et les dieux agraires, ainsi que les déesses locales du type "Vénus" sont examinées, ainsi que Dionysos (avec thiase, ménade, satyres, etc.) dont la présence retient l'attention par sa fréquence et sa forte implantation. Les personnifications, en particulier Tyché, garante de prospérité et de fortune, et la Victoire, méritent aussi quelques commentaires, tout comme Déméter et Triptolème qui renvoient certainement au modèle éleusinien. On n'oubliera pas les divinités armées ou courotrophes.

6 Le cinquième et dernier chapitre de la seconde partie, sur les ateliers, les sculpteurs et les convergences qui émergent par ce biais, est l'occasion de se demander qui produit ces reliefs, avec quels moyens, pour quels publics et quelles finalités, avec quels modèles. C'est aussi une manière d'aborder les questions de circulation et de transmission de techniques, savoirs, styles, etc. Dans certains cas, comme à Baalbek, 
immense chantier impérial, des ateliers étaient établis sur place et travaillaient en collaboration, parfois sur une même pièce. Ainsi, montre l'Auteur (p. 296), sur l'autel monumental de Baalbek, on trouve des traces de deux styles différents («plat» et « rude »), ce qui complique ultérieurement la datation de son exécution. En matière de convergences, certaines œuvres de Niha et d'ailleurs présentent des éléments caractéristiques des sculptures de la Syrie intérieure (Palmyre et Doura-Europos). Certains rapprochements avec la statuaire de Jordanie sont possibles, mais aussi avec les ateliers microasiatiques pour les reliefs en marbre de la côte (Tyr et Sidon), signe d'une pluralité de "convergences» que la rareté des signatures de sculpteurs ou d'architectes ne permet guère de cerner de plus près. Du reste, aucune de ces signatures ne comporte d'ethnique, ce qui induit Z. Fani Alpi à voir dans ces artisans/ artistes des « indigènes ".

Une brève conclusion (p. 302-303) rassemble les acquis d'une importante enquête qui, à travers les reliefs, montre bien l'ancrage persistant des traditions locales, parfaitement aptes à s'adapter à un environnement romain, à se l'approprier et à le mettre au service d'un discours figuré propre à cette région. Les indices de grande maîtrise artistique, analogue à celle que manifestent les productions julio-claudiennes dans tout l'Empire, sont nombreux, mais ils ne conduisent pas à renoncer, localement, à la frontalité des dieux et ils n'empêchent nullement la valorisation des figures divines du territoire, comme la triade héliopolitaine. La richesse des cultes régionaux est mise au service de la grandeur de Rome. Les dieux de la koinè gréco-romaine, comme Dionysos, Hermès, Aphrodite, Héraclès et bien entendu Zeus, sont donc à la fois ces figures partagées qui circulent dans toute la Méditerranée et des «interprétations » locales ou régionales traduites dans un langage figuré prestigieux, plastique, au fort potentiel narratif et visuel, qui inscrit le Liban romain dans un cadre culturel valorisant.

Il faut savoir gré à Zeina Fani Alpi d'avoir porté à la connaissance de la communauté scientifique et si soigneusement analysé un corpus ample et complexe, fondamental pour l'étude de l'histoire culturelle et religieuse de la Phénicie d'époque romaine. L'index qui clôture le volume favorisera ultérieurement les approfondissements, comme par exemple une étude systématique de la présence de Dionysos/Bacchus dans cette région et des significations qui s'y attachent en termes de cultes.

\section{AUTEURS}

\section{CORINNE BONNET}

Université Toulouse - Jean Jaurès, PLH-ERASME, cbonnet(at)univ-tlse2.fr 